32. Steinbuch $\mathrm{Y}$. Italian nurse with coronavirus kills herself over fear of infecting others. New York Post.2020 Mar 25[cited 2020 Apr 26]. Available from: https://nypost.com/2020/03/25/italian-nurse-with-coronaviruskills-herself-amid-fears-of-infecting-others/
33. Rahn W. German state finance minister Thomas Schäfer found dead. DW.com. 2020 Mar 29[cited 2020 Apr 26]. Available from: https://www. dw.com/en/german-state-finance-minister-thomas-schäfer-founddead/a-52948976

\title{
Clinical ethics during Covid-19: Plan for the whole health ecosystem
}

\section{CHETANYA MALIK, TIMOTHY LAUX, YOGESH JAIN}

\begin{abstract}
During a pandemic, narrowing ethics into silos such as clinical and public health does not help the cause of ethics, which often gets neglected in desperate times. Our response to a recently published article in this journal, tries to take this discussion forward. Keeping medical ethics at the centre of our response to the Covid-19 pandemic would benefit healthcare systems at all levels. This would also help us be prepared for future pandemics. Strengthening healthcare systems would also provide an opportunity to improve non-Covid care.
\end{abstract}

Keywords: Covid-19, health ecosystem, non-Covid care, patient autonomy, healthcare worker welfare, pandemic as opportunity

The article "Clinical ethics during the Covid-19 pandemic: Missing the trees for the forest" by Gopichandran (1) highlights key ethical issues in clinical practice during the surge of the Covid-19 pandemic. It discusses several issues related to both Covid and non-Covid care. The author makes observations relevant to the ethics of care in hospitals. He brings to our attention several dilemmas faced by healthcare workers, especially doctors. A similar discussion in relation to non-Covid care is also a part of the narrative. We wish to underscore a few key points raised in the article and add to the discussion around clinical ethics during this pandemic.

\section{The "missing the trees for the forest" analogy}

Our experience has been that strategies at all levels of the health "ecosystem" (from community work to tertiary care) constitute a continuum rather than the "either-or" dichotomy presented in this paper. Tracing the contacts of a Covid-19 positive patient who subsequently requires hospitalisation is an apt illustration of this point. The relevant ethical principles would apply

\footnotetext{
Authors: Chetanya Malik (drchetanyamalik86@gmail.com), Junior Faculty, Jan Swasthya Sahyog, Village Ganiyari, Dist. Bilaspur, Chattisgarh INDIA Timothy Laux (laux.timothy@gmail.com), Junior Faculty, Jan Swasthya Sahyog, Village Ganiyari, Dist. Bilaspur, Chattisgarh INDIA; Yogesh Jain (yogeshjain.jssbilaspur@gmail.com), Senior Faculty, Jan Swasthya Sahyog Village Ganiyari, Dist. Bilaspur, Chattisgarh INDIA.

To cite: Malik C, Laux T, Jain Y. Clinical ethics during Covid-19: Plan for the whole health ecosystem. Indian J Med Ethics. 2020 Jul-Sep;5(3)NS: 187-9 DOI: 10.20529/JME.2020.065

Published online on June 3,2020

(c) Indian Journal of Medical Ethics 2020
}

to the teams working on surveillance, contact tracing, and transportation, and not solely to the inpatient team. We would argue, both as a matter of principle and practice, that public health strategies and clinical care must work in tandem; and hence not just the forest and trees, but the shrubs and the entire ecosystem need attention. Recalibration between various parts of the healthcare ecosystem should not be at the expense of ethics as a principle, be it in the hospital or in the community.

\section{Ethical dilemmas with regard to patient autonomy}

When deciding on treatment options in a pandemic, whether new therapies or established options, patient autonomy is compromised given the strict isolation required. So too, is family participation in the care of their sick loved ones. While the caregiver's distress is moral, the patient's distress is more fundamental. Lack of family support in general for routine care, emotional support, and for sharing in important life altering decisions like intensive care and ventilator support gives rise to immense stress and impacts the patient's autonomy

\section{Lack of palliative care and mental health support}

Management protocols need to include palliative care options where relieving physical and mental distress and respecting the patient's autonomy are paramount. Similarly, mental health support for all those admitted into isolation and quarantine facilities, irrespective of pre-existing mental health illness, should be part of management. A mental health nurse, counselors or psychiatrist, depending on the level of care, will be helpful in this regard. Both these components should not be on an "if and when" basis, but be part of the standard management and non-negotiable rights of those with Covid-19 disease.

\section{Healthcare worker welfare}

Healthcare worker welfare extends far beyond the dilemmas inpatient providers face in the ward, as outlined in the article (1). The welfare of healthcare staff, working in hospitals at all levels (doctors, nurses, orderlies, sanitation staff etc), and outside (surveillance team, quarantine centres, Accredited Social Health Activists (ASHA) is of vital importance. Much needs to be done beyond hailing them as "corona warriors" (2). Stigma against health workers should be addressed through both state and community interventions. Education of healthcare workers about various aspects of the disease, the risks involved at each 
level of care, appropriate use of personal protective equipment (PPE), infection control measures and mental health issues will help. For those working in Covid hospitals, regular symptom checks, availability of appropriate PPE, hand washing facilities, prioritised testing and adequate quarantine facilities must be provided. Such practices should be seen as part of ethical clinical practice both to reduce infection and transmission of infection; and to ensure the rights of healthcare workers as envisaged by the World Health Organization (3). These practices need to slowly move to other health facilities given the impending spread and threat of infection even in non-Covid facilities.

\section{Dilemmas in relation to experimental therapies}

Hydroxychloroquine is a drug used for autoimmune disorders and is, at best, of unproven benefit in Covid-19 infection (4). Nonetheless, it has been recommended by the Indian Council of Medical Research (ICMR) (5) for prophylaxis for healthcare workers. This recommendation has been taken up as mandatory by health departments in various states, and is being provided to all healthcare workers who are using it as a prophylactic agent. This is an example of a drug being used more in the hope of benefit, than any real, observed benefit in clinical trials. Such behaviour compromises clinical ethics on various fronts including: (i) lack of autonomy for those using it as a prophylactic, as what should have been, at best, advisory has changed into a government order; (ii) a false sense of security given the lack of evidence, till now, of its benefit; (iii) non-availability for many patients needing these drugs for other illnesses (rheumatoid arthritis) where it has clear benefit.

\section{Non-Covid healthcare delivery}

Routine antenatal care, childbirth and immunisation service delivery have decreased as per data released by the National Health Mission (6).The tuberculosis care programme and non-communicable disease care have taken a hit in various parts of the country with lockdown in place. This is due to severe restrictions on travel, focus on Covid-related activities, and poor primary healthcare services in large parts of the country, especially rural India (6). With rising cases of Covid-19 and as the lockdown is eased, pressure on healthcare facilities and care providers will rise. While staff is being diverted for Covid-19 care, those engaged in non-Covid healthcare delivery would need support at both administrative and personal levels. The administration would need to strike a balance between the two, instead of compensating one at the cost of other, which may end up severely compromising care for those requiring non-Covid care. Daily media projections of the number of Covid-19 related deaths prevented by the various lockdowns are incomplete without data about the number of preventable, non-Covid-19 related deaths that have occurred during the same period. While such data will likely not be forthcoming, acknowledging its absence is critical.

\section{Covid-19 as opportunity for a different future}

While it is only natural for public health professionals and clinicians to be exclusively focused on the global pandemic, we foresee one danger with this (justifiable) myopia. All attention is focused on the present and its problems. How can we use the response to Covid-19 to chip away at some of the least desirable aspects of healthcare and public health in India? Covid-19 can create a space for both public health and clinical professionals to plan a strong health system which can respond adequately to future disease outbreaks. Perhaps surveillance systems put into place during this pandemic can be transitioned to provide more accurate, more robust data about other infectious diseases in the future. Perhaps screening, diagnosis and treatment of non-communicable diseases like diabetes and hypertension, especially in rural areas, will help in reducing suffering among vulnerable groups due to both Covid-19 and non-Covid causes. Perhaps enlarging and strengthening government hospitals can allow for a long term response to Covid-19 in the present, and better non-Covid-19 care in the future. A fully functional intensive care unit with ventilators and a regular oxygen supply is still a pipe-dream for many district hospitals in the country. Perhaps new laboratory infrastructure can remain in place in the decades to come. Enzyme linked assay readers and CT machines can be envisaged by administrations with foresight beyond the present pandemic, as an investment for the future. Such thinking leading to action is welcome. This would mutually benefit clinicians delivering care to a patient and public health professionals in delivering results at a population level. While Covid-19 is a disaster, it is also an opportunity that will likely not come again during our lifetime.

\section{Conflict of interest: No conflict of interest declared.}

Disclaimer: The views expressed in this article are the personal opinions of the authors and do not represent the views of Jan Swasthya Sahyog.

\section{References}

1. Gopichandran V.Clinical ethics during the Covid-19 pandemic: Missing the trees for the forest. Indian J Med Ethics. Published online on 2020 Apr 30 [cited 2020 May 20]. Available from: https://ijme.in/articles/ clinical-ethics-during-the-covid-19-pandemic-missing-the-trees-forthe-forest/\#

2. Ministry of Defence, Govt of India. Frontline warriors salute Corona Warriors and VOW to continue supporting the fight [Press release]. New Delhi: MoD; 2020 May 1 [cited 2020 May 20]. Available from: https://pib. gov.in/PressReleseDetail.aspx?PRID=1610749

3. World Health Organization. Coronavirus disease (covid-19) outbreak: rights, roles and responsibilities of health workers, including key considerations for occupational safety and health. Geneva:WHO; 2020 Mar 18[cited 2020 May 20]. Available from: https://www.who.int/docs/ default-source/coronaviruse/who-rights-roles-respon-hw-covid-19. pdf?sfvrsn=bcabd401_0

4. Geleris J, Sun Y, Platt J, Zucker J, Baldwin M, Hripcsak J, et al. Observational study of hydroxychloroquine in hospitalized patients with Covid-19.N Engl J Med. 2020 May 7;.NEJMoa2012410.doi: 10.1056/ NEJMoa2012410. Epub ahead of print.

5. Indian Council of Medical Research. Advisory on the use of hydroxy- 
chloroquine as prophylaxis for SARS-CoV-2 infection. New Delhi: ICMR 2020 Mar 22 [cited 2020 May 20]. Available from: https://www.mohfw. gov.in/pdf/AdvisoryontheuseofHydroxychloroquinasprophylaxis forSARSCoV2infection.pdf
6. Rukmini S. How covid-19 response disrupted health services in rural India. Livemint. 2020 Apr 27[cited 2020 May 20]. Available from: https:// www.livemint.com/news/india/how-covid-19-response-disruptedhealth-services-in-rural-india-11587713155817.html

\section{To comfort always: Are we ignoring this duty in Covid protocols?}

\section{MR RAJAGOPAL}

\begin{abstract}
Covid-19 is an amplifier of serious physical suffering and emotional trauma, which together could be all-consuming. It is important for health systems to go beyond methods of prevention and treatment, and focus on the palliation of suffering, and to systematically integrate palliative care into Covid-19 management.
\end{abstract}

Further, in cases where the triage process indicates poor chances of survival, it is particularly important to respect autonomy by honest and sensitive disclosure of prognosis, and to jointly arrive at goals of care. Hooking every dying person to a ventilator would violate the ethical principles of beneficence and non-maleficence. It is also important to ensure at least electronic communication between the patient and family members.

Keywords: Covid-19, palliative care, end of life care, isolation, quarantine, intensive care, ethics of intubation, consent

Dr Peter Piot is a Belgian virologist. He was one of the discoverers of the Ebola virus. He has spent decades working on HIV/AIDS and is a special advisor to European Commission President Ursula von der Leyen.

And he got Covid-19 at the age of 71. In an interview, much of what he talks about is not the virus; it is about what he experienced (1).

On 19 March, I suddenly had a high fever and a stabbing headache. My skull and hair felt very painful, which was bizarre" he says. "It turned out I had severe oxygen deficiency... I was concerned I would be put on a ventilator immediately because I had seen publications showing it increases your chance of dying.... At home, I cried for a long time. I also slept badly for a while.... I realize this one will

Author: MR Rajagopal (chairman@palliumindia.org), Founder Chairman, Pallium India, Thiruvananthapuram, Kerala, 695009 INDIA

To cite: Rajagopal MR. To comfort always: Are we ignoring this duty in Covid protocols? Indian J Med Ethics. 2020 Jul-Sep; 5(3) NS: 189-91 DOI: 10.20529/ IJME.2020.071

Published online on June 30, 2020.

Manuscript Editor: Sandhya Srinivasan

(c) Indian Journal of Medical Ethics 2020 change my life, despite the confrontational experiences I've had with viruses before. I feel more vulnerable.

What $\mathrm{Dr}$ Piot describes is suffering. The pain, the breathlessness, the fear, the loneliness.

But healthcare systems around the world ignore suffering. They have responded to Covid-19 in the disease-focused way that is familiar to them - with the measures of prevention (lock-down, social distancing, quarantine of the affected and "suspected"), diagnosis (testing as many as possible for early detection) and treatment aimed at organ dysfunction, as they do not know of a cure. Rightly so, as the disease needs attention, the virus needs attention. But it is unethical to forget the human being and to ignore suffering. Too little is discussed and done about preventing, identifying and managing the symptom burden, the psycho-social consequences of isolation, the post-traumatic stress disorder or the pathological grief of the bereaved, from whom their loved one was snatched away in an instant, sometimes never to be seen again, denied the rituals that are important in their belief systems and, not infrequently, even one last look at the body.

What indeed is the duty of care of the healthcare provider? According to the Indian Council of Medical Research, it is to "mitigate suffering. It is to cure sometimes, relieve often and comfort always. There exists no exception to this rule."(2).

The suffering in Covid-19 is caused both by the disease and by social and governmental reactions to it, as with the lockdown.

In the physical domain, symptoms can be mild, flu-like feelings for some; and for others, intense suffering that they have never known before. For those with serious illness - as it progresses, often the lungs get affected, causing breathlessness. Intense breathlessness is one of the most difficult symptoms to bear - the sensation that one is unable to take in enough air leads to the compounding of that agony by the feeling, "Am I going to die without being able to take in any air?" As the disease advances, many people get delirious. Ordinarily, delirium is not taken seriously by those around the patient. But the abnormal feeling that it causes can be hard to bear, especially when one gets hallucinations. How can one get a moment of peace when one is afraid of demons waiting around, ready to attack any moment? Or when the nurse who walks towards one seems to have fangs and to carry a lethal weapon?

We of the medical system are best at treating what we can see clearly on an imaging screen and when that can be excised, 